Encontros Bibli: revista eletrônica de biblioteconomia e ciência da informação, v. 21, n. 47, p. 51-66, set./dez., 2016. ISSN 1518-2924. DOI: 10.5007/1518-2924.2016v21n47p51

ARTIGO

Recebido em:

20/05/2016

Aceito em:

26/07/2016

\title{
La Bibliometría, Informetría, Cienciometría y otras "Metrías" en el Brasil
}

\author{
Bibliometrics, informetrics, scientometrics and other "Metric" in \\ Brazil
}

Ruben URBIZAGASTEGUI

Universidade da California em Riverside - ruben@ucr.edu

\section{Resumen}

Analiza las características demográficas de la literatura publicada sobre "estudios bibliométricos" producidos por autores brasileños y extranjeros en el Brasil desde 1973 hasta 2012. Se identifican los tipos de documentos, las revistas y los congresos más utilizados para comunicar los resultados de las investigaciones y los autores más productivos. Se encontraron 2,300 trabajos publicados por 3,320 autores hasta diciembre de 2012. Sobresalen los artículos publicados en revistas académicas con $60 \%$ y los trabajos presentados en eventos, congresos, etc. con $36.5 \%$. Predominan los documentos publicados en portugués con $87 \%$. Las revistas con el mayor número de artículos publicados sobre estos temas son Ciência da Informação, Scientometrics, Encontros Bibli y Perspectivas em Ciência da Informação.

Palabras-clave: Bibliometría. Informetría. Cienciometría. Brasil.

\begin{abstract}
Analyze the demographics of the published literature on "bibliometric studies" produced by Brazilian and foreign authors in Brazil from 1973 to 2012. Types of documents, journals and congresses most used to communicate the results of investigations are analyzed. The most productive authors were identified. 2,300 papers published until December 2012 by 3,320 authors were found. More common are papers published in academic journals $(60 \%)$ and papers presented at congresses and conferences (36.5\%). Predominately, documents area published in Portuguese (87\%). The journals with the largest number of published papers are Ciência da Informação, Scientometrics, Encontros Bibli and Perspectivas em Ciência da Informação.
\end{abstract}

Keywords: Bibliometrics. Informetrics. Scientometrics. Brazil.

\section{INTRODUCCIÓN}


La comunicación a través de las revistas académicas es la forma más utilizada por los científicos para difundir los resultados de sus investigaciones. En general, las revistas más prestigiosas en un determinado campo son las que reciben un mayor número de artículos buscando publicación. Pero en el campo científico no basta con la publicación también se tiene que buscar la visibilidad del documento publicado. Esa literatura publicada es recogida e indizada en bases de datos bibliográficas especializadas, aunque por múltiples razones esas bases de datos nunca son exhaustivas ni consiguen indizar todo lo publicado en un país. La recolección de artículos es selectiva, ya que siguen sus propios criterios e intereses. Por ejemplo, la búsqueda por el tópico "bibliometrics" y la dirección postal "Brazil" en el Web of Science produce 167 referencias y en PubMed se recuperan solo 318 referencias. Al respecto algunos investigadores comentan que la representatividad de la base de datos del ISI en determinados campos como la física y la biología es poco cuestionada por la comunidad científica por ser la más completa y exhaustiva, pero no ocurre lo mismo con las disciplinas de las ciencias sociales y humanidades que, por su naturaleza, son menos internacionalizadas. Según Santos (2003, p. 132) "muchas de las publicaciones más respetadas y "nobles" del área (como las europeas), cuyas revistas no tienen al inglés como la lengua materna, no son indizadas. Esto hace que el mundo académico vea al Social Sciences Citation Index, con algunas reservas, teniendo en cuenta su baja representatividad y recomendando su uso con mucha cautela". Yo diría más bien desconfianza.

Esa desconfianza en relación a la recopilación e indización parcializada de la producción académica brasileña en el campo de la bibliotecología y ciencia de la información y más específicamente en campo de la bibliometría, informetría, cienciometría y otras metrías, por bases de datos bibliográficas "internacionales" impulsó a la creación de una base de datos específica sobre este asunto y al análisis posterior de sus resultados.

El objetivo de este artículo es analizar la literatura publicada sobre las metrías (bibliometría, informetría, cienciometría, patentometría, arquivometría, etc.) en el Brasil. El periodo escogido se extiende desde los primeros trabajos publicados en 1973 hasta diciembre de 2012, un periodo extenso como para esperar que la literatura publicada se acumule y sedimente. El autor de este artículo elaboró una base de datos bibliográfica de la literatura publicada sobre bibliometría en el Brasil en Endnote 5.0. Aprovechando esta base de datos es oportuno analizar los diferentes aspectos que presenta esta literatura, especialmente el estudio de las características demográficas de la literatura publicada focalizándose en las siguientes cuestiones:

a) ¿Cuáles son los vehículos de comunicación más usados para informar los resultados de la investigación?

b) ¿Cuáles son los idiomas empleados para comunicar esta literatura?

c) ¿Cuál es la forma de dispersión de esta literatura? ¿Es posible identificar un núcleo de revistas o eventos dedicados al asunto? ¿Existen revistas especializadas en bibliometría en el país?

d) ¿Existe una elite de productores identificables y esta elite es responsable de la mitad de la literatura publicada? ¿Estos autores colaboran entre sí? ¿Cuál es su coeficiente de colaboración? ¿Y cuál es su tasa de productividad?

Para lograr el objetivo propuesto, este artículo está organizado en seis partes. En la primera se presenta una introducción al tema, se detalla el problema y se formulan las preguntas de investigación. En la segunda se revisa la literatura publicada sobre este asunto. En la tercera se describe la metodología empleada con énfasis en la forma de recolección de los datos y la forma de medición de los mismos. En la cuarta parte se exponen los resultados. En la quinta se proponen las 
conclusiones y discusiones de los resultados obtenidos. Por último se presenta la bibliografía revisada para la elaboración de este trabajo.

\section{REVISIÓN DE LA LITERATURA}

Hasta donde es del conocimiento de los autores de este artículo ningún investigador ha analizado la literatura brasileña sobre bibliometría en un periodo tan extenso y cubriendo todos los campos donde se exploran las aplicaciones bibliométricas en el país. El análisis de esta literatura ha sido realizado en periodos más cortos y centrados en campos especializados. Por ejemplo, Urbizagástegui (1984) analizó la producción científica brasileña en bibliometría entre 1972 y 1983 en el campo de la ciencia de la información encontrando 78 documentos publicados. Sus resultados mostraron que la ley de Bradford fue el tema principal de la producción intelectual hasta los años 1980, lo que podría explicarse por las aplicaciones prácticas inmediatas para la elaboración de listas básicas de revistas dedicadas a la formación de colecciones en las bibliotecas y centros de documentación existentes en el país. Casi 20 años después Vanz (2003) analizó los artículos sobre bibliometría publicadas en la revista Ciência da Informação en el período de 1972 a 2002. La autora señaló que existen pocas publicaciones sobre bibliometría en el país, aunque encontró que existe un interés creciente sobre este asunto desde finales de los años 1990. Aparentemente también "hay un estudio [de] Mugnaini y colegas (2004), que analizaron los indicadores de publicación que muestra el aumento significativo de la producción científica brasileña en bibliometría en los últimos 20 años, así como su internacionalización. También llegaron a la conclusión que existe una concentración de estos investigadores en la región sureste y que hay una ampliación de la alianza de investigadores brasileños con investigadores de otros países principalmente Estados Unidos y Europa" (Citado por MATOS; JOB, 2008, p. 50).

Poco después Machado \& Pinto (2005) estudiaron la producción científica en bibliometría a partir de los artículos publicados en cinco revistas del campo de biblioteconomía y ciencia de la información brasileñas, en el periodo de 1990 a 2004 identificando apenas 27 artículos publicados en ese periodo. Analizaron la producción anual, el tipo de publicación, la categoría y la afiliación institucional de los autores, el idioma y los asuntos estudiados en los artículos. Indicaron que la producción científica se concentra en el sudeste del país $(68,75 \%)$, y que las universidades (58\%) son las mayores productoras. Nuevamente Machado (2007) analizó los asuntos estudiados de 1990 a 2005 en las mismas cinco revistas brasileñas de biblioteconomía y ciencia de la información estudiadas anteriormente. Identificó 31 documentos publicados en ese periodo, 21 de los cuales fueron publicados por 19 autores brasileños y 10 documentos fueron publicados por autores extranjeros. Al año siguiente Fontes (2008) analizó la literatura sobre los estudios métricos realizados en el Brasil en 10 revistas electrónicas de bibliotecología y ciencias de la información desde sus inicios hasta el año 2007. Para la recolección de los datos utilizó la base de datos Dedalus de la Universidad de São Paulo y SciELO CAPES. Identificó la productividad de los autores, los tipos de autoría y las áreas de aplicación. Encontró que la mayoría de las revistas están vinculadas a los cursos de postgrado del campo de la $\mathrm{BCI}$, también encontró que hay dispersión de la productividad de los autores y que la mayoría de estos prefieren trabajar en colaboración. Ese mismo año Pinheiro e Silva (2008) realizaron un análisis de la producción de tesis sobre los estudios métricos en el Brasil de 1972 al 2008. El objetivo central de este texto fue hacer una revisión histórica del desarrollo de la bibliometría, particularmente su desarrollo en el Instituto Brasileño de Información en Ciencia y Tecnología (IBICT), pero también la producción de otras instituciones. En el periodo estudiado identificaron 57 tesis producidas sobre este asunto. También 
Matos e Job (2008) analizaron la producción científica de autores brasileños vinculados al campo de la ciencia de la información que investigaron sobre bibliometría, o bien utilizaron las técnicas bibliométricas como método y publicaron los resultados de sus investigaciones en la revista Scientometrics entre 1978 y 2006. Encontraron 34 referencias de artículos publicados por 34 autores brasileños la mayoría de ellos prefiriendo la autoría compartida con autores nacionales. Luego serán Meneghini e Packer (2010) quienes recogieron los artículos sobre cienciometría y bibliometría producidos en el país en el periodo de 1990 a 2006, pero usaron para la recolección de los datos el Web of Science, Google Scholar, SciELO Brasil y la Plataflorma Lattes. En ese periodo encontraron un total de 197 documentos de los cuales $78 \%$ fueron publicados en 57 revistas brasileñas y $22 \%$ en 13 revistas extranjeras. Afirmaron que las publicaciones en cientometría y bibliometría producidas por autores brasileños crecieron exponencialmente 13 veces en la base de datos del Web of Science, y 19,5 veces en el Google Scholar. Atribuyeron este crecimiento de la literatura, por un lado, al desarrollo del internet y a la disponibilidad de publicaciones y datos estadísticos en bases de datos como Web of Science, Google Scholar y SciELO. Por otro lado, al reconocimiento de la utilidad de la bibliometría y cienciometría en la evaluación de la administración de la ciencia, tecnología e innovaciones. Al año siguiente Araújo e Alvarenga (2011) analizaron la utilización de las técnicas bibliométricas en las tesis y disertaciones de los cursos de postgrado en el Brasil producidos entre 1987 y 2007 . Para la recopilación de los datos usaron el Banco de Tesis de CAPES y el término "bibliometría" en el campo de las palabras-clave, resúmenes y títulos de las tesis. Encontraron 82 tesis en el periodo investigado y 23 instituciones de enseñanza superior donde ocurrieron la defensa de las tesis y disertaciones. Ese mismo año Azambuja (2011) analizó el uso de métodos bibliométricos en los artículos publicados por 8 revistas brasileñas del campo de la bibliotecología y ciencias de la información en el periodo de 2006 a 2011. Encontró apenas 33 artículos, con una media de publicación de 5.6 artículos por año. También Lima; Soares e Oliveira (2011) analizaron la producción científica en el tema "estudios métricos" indizados en la Base de Datos Referenciales de Artículos de Revistas en Ciencia de la Información (BRAPCI) en el periodo de 1991 hasta marzo del 2011. Encontraron un total de 151 artículos y 203 investigadores productores en esta área, pero están más preocupados con estudiar la productividad, y las redes de co-autorías institucionales. Igualmente, Grácio e Oliveira (2012) realizaron un estudio diacrónico de la contribución científica sobre el asunto "estudios métricos" para la ciencia "mainstream" analizando las revistas indizadas en la base SCOPUS en el periodo de 1984 a 2012 e identificando a los autores brasileños más productivos, sus redes de colaboración y sus niveles de citación. Después de los respectivos filtrados la población estudiada fue reducida a 263 artículos producidos por 649 investigadores diferentes. Fueron identificados 31 autores brasileños tipificados como los más productivos con 3 o más artículos publicados en 113 revistas diferentes. Finalmente Leta (2012) hizo un recuento del desarrollo de la cienciometría en el Brasil enfatizando la necesidad de resolver tres viejas cuestiones relacionadas con la bibliometría nacional: primero recobrar y re-estructurar la identidad del campo; segundo recobrar las facetas básicas de la cienciometría; y tercero eliminar las falsas concepciones sobre las metrías.

La Tabla 1 (ver Anexo A) ofrece un cuadro resumido de autores, fuentes utilizadas para la recolección de los datos, los periodos cubiertos y los resultados obtenidos en la literatura revisada anteriormente. Esas investigaciones están centradas en el campo de la bibliotecología y ciencias de la información en periodos cortos de tiempo. En ninguno de esos documentos existe la intención de analizar la literatura sobre bibliometría publicada en el país desde sus inicios y abarcando todo lo publicado en el país y/o fuera de sus fronteras por autores brasileños o extranjeros interesados en publicar en el país. Tampoco parece existir interés en mapear todos 
los campos científicos posibles en los cuales se están explorando las técnicas bibliométricas ni en producir los indicadores correspondientes, a pesar de ser evidente que "el interés en bibliometría ha aumentado considerablemente desde la década de 1970 hasta la actualidad, ya sea debido al aumento de los cursos de postgrado en el país [...], ya sea por el crecimiento cuantitativo y cualitativo de las revistas científicas. Pero este crecimiento se ha producido en todas las ciencias" (MATTOS; JOB, 2008). Por esa razón, este trabajo pretende también llenar ese vacío y marcar pautas para futuras investigaciones de este tipo.

\section{MATERIAL Y MÉTODOS}

Como unidades de análisis fueron tomados cada uno de los artículos publicados en revistas académicas, capítulos de libros, y trabajos presentados en congresos que trataron algunos de los aspectos de los estudios métricos (bibliometría, cienciometría, informetría, etc.) o las aplicaciones de estas técnicas en una disciplina o sub-campo determinado en el Brasil. Se excluyeron libros, tesis, monografías y literatura gris por dos razones. Primero porque los libros comienzan como artículos publicados en revistas especializadas; y segundo porque las tesis, monografías y literatura gris no son indexadas en varias de las bases de datos bibliográficas consultadas. El periodo cubierto va desde los primeros trabajos publicados hasta diciembre del 2012.

Para recolectar los datos fueron efectuadas búsquedas usando los términos listados en el Anexo B en sus diferentes acepciones idiomáticas (inglés, francés, alemán, portugués, español, etc.) y en múltiples combinaciones booleanas en los títulos, palabras clave y resúmenes de las bases de datos bibliográficas y portales de bibliotecas listadas en el Anexo C.

Las referencias identificadas fueron después exportadas a EndNote X5 para la elaboración de una base de datos sobre el asunto. Posteriormente fue realizada una minuciosa lectura de cada uno de los documentos identificados en la búsqueda, dedicando especial atención a cada referencia efectuada en el documento leído. Después cada referencia relativa al asunto de investigación era confrontada con la base de datos e incorporada si no había sido identificada en la búsqueda anterior. Lógicamente las referencias duplicadas fueron eliminadas, manteniéndose solamente una referencia no repetida. La identificación de la pertinencia del documento fue una tarea ardua y difícil, porque con mucha frecuencia las palabras clave fueron insuficientes y a veces falsos indicativos. Para identificar a los autores brasileños se analizó la afiliación institucional de cada autor identificado en cada referencia recuperada. Muchas veces fue necesario consultar la Plataforma Lattes para realizar esta verificación o buscar los "currículo vitae" de los autores, y en no pocas situaciones, se realizaron minería de textos. La nacionalidad y la ligación institucional fueron también indagadas a través de mensajes personales vía el correo electrónico del investigador identificado. Con toda esa estrategia y la lectura minuciosa de muchos de los artículos identificados fue producida la base de datos (que sirve de base al objeto de esta investigación) que contiene referencias de artículos de revistas, capítulos de libros, comunicaciones presentadas en congresos, una que otra nota editorial y cartas enviadas a los editores de las revistas académicas. Esta base de datos especializada ha estado en construcción permanente por un periodo de más de dos años.

El análisis de los datos recolectados fue realizado con la ayuda de Microsoft Excel y SPSS (versión 17.0 para Windows). En estos softwares se realizaron las estadísticas descriptivas e inferenciales pertinentes. Para identificar las revistas y eventos más utilizados para comunicar los resultados de las investigaciones se usó el método de división zonal de la Ley de Bradford propuesto por Egghe (1990). Cuando las "fuentes" son organizadas en orden de productividad decreciente: 
$\mathrm{y}_{\mathrm{m}}=$ es el no. de ítems en la fuente más productiva (fuentes en el rango 1)

$R(r)$ = es el número acumulado de ítems producidas por las fuentes de rango 1, 2, 3, ...r.

a y b son constantes que aparecen en la Ley de Leimkuhler:

$\mathrm{R}(\mathrm{r})=\mathrm{a} \log (1+\mathrm{b} r)$

donde

$r=1,2,3, \ldots \ldots$

$a=\frac{y_{o}}{\log k}$

$b=\frac{k-1}{r_{o}}$

$k=\left(e^{y} y_{m}\right)^{1 / p}$

donde e es el número de Euler $(\mathrm{e}=1.781)$. De aquí se sigue que:

$\gamma_{o}=\gamma^{2} \log k$

$r_{o}=(k-1) \gamma_{m}$

Para identificar la élite de los autores más productivos se usó la "ley de la raíz cuadrada" de Price (1963; 1965; 1969; 1971; 1975). Según esta ley, si k representa el número total de contribuyentes en una disciplina, $\sqrt{N}$ representaría la élite del campo estudiado y esta élite generaría la mitad de las contribuciones publicadas. Para cada uno de estos autores se estimó también su respectivo "coeficiente de colaboración" (CC) (AJIFERUKE; BURELL; TAGUE, 1988). Este CC está basado en la idea de la atribución fraccional de la producción de documentos y puede ser expresada como:

$C C=1-E\left[\frac{1}{X}\right]$

$C C=1-\sum\left(\frac{1}{j}\right) P(x=j)$

y su muestra estimada es

$C C=1-\frac{f_{1}+\left(\frac{1}{2}\right) f_{2}+\ldots+\left(\frac{1}{k}\right) f_{k}}{N}$ 
$C C=1-\frac{\sum_{j=1}^{k}\left(\frac{1}{j}\right) f_{j}}{N}$

donde

$f_{j}=$ es el número de artículos de investigación con j-autores múltiples publicados en una disciplina durante un determinado periodo

$\mathrm{N}$ = es el número total de artículos de investigación publicados en una disciplina durante un determinado periodo; $y$

$\mathrm{k}=$ el mayor número de autores por artículo en una disciplina

También se estimó la tasa de producción (TP) de cada autor del grupo que conforma la elite de productores en bibliometría en el Brasil. Esta tasa de producción no es más que la división del número de total de documentos producidos entre el número de años transcurridos desde el año de publicación del primer documento hasta el año de publicación del último documento de cada autor analizado.

\section{RESULTADOS}

Se encontraron 2300 trabajos publicados en el Brasil y en otros países por 3,320 autores brasileños y extranjeros que eligieron revistas o eventos brasileños para comunicar los resultados de sus investigaciones. La forma de comunicación predominante son los artículos publicados en revistas académicas (60\%), luego las ponencias presentadas en eventos (36.5\%), en menor frecuencia los capítulos de libros $(2.5 \%)$, las notas editoriales (1.13\%) y finalmente las cartas enviadas a los editores de revistas $(0.13 \%)$ (Ver Tabla 2$)$.

Tabla 2: Tipos de documentos publicados según los idiomas.

\begin{tabular}{lrccccr}
\hline Idiomas & Artículos & $\begin{array}{c}\text { Ponencias } \\
\text { congresos }\end{array}$ & $\begin{array}{c}\text { Cap. } \\
\text { libros }\end{array}$ & $\begin{array}{c}\text { Nota } \\
\text { editorial }\end{array}$ & $\begin{array}{r}\text { Cartas } \\
\text { al editor }\end{array}$ & TOTAL \\
\hline Portugués & 1118 & 809 & 52 & 19 & -- & 1998 \\
Inglés & 179 & -- & -- & 1 & 1 & 181 \\
Español & 46 & 6 & 2 & -- & -- & 54 \\
Francés & 2 & 25 & 4 & -- & - & 31 \\
P/E/I & 21 & -- & -- & 3 & -- & 24 \\
P/I & 7 & -- & -- & 3 & 2 & 12 \\
\hline TOTAL & $\mathbf{1 3 7 3}$ & $\mathbf{8 4 0}$ & $\mathbf{5 8}$ & $\mathbf{2 6}$ & $\mathbf{3}$ & $\mathbf{2 3 0 0}$ \\
\hline
\end{tabular}

Fuente: datos de la investigación. Notas: $\mathrm{P} / \mathrm{E} / \mathrm{I}=$ Portugués/Español/Inglés; $\mathrm{P} / \mathrm{I}=$ Portugués/Inglés.

Para identificar las revistas más utilizadas para comunicar los resultados de las investigaciones se usó el método de división zonal de la ley de Bradford propuesto por Egghe (1990). Esta división se estimó con $\mathrm{A}=1402 ; \mathrm{P}=3$ y T $=462$ dando como resultado yo $=350.5 ; \mathrm{k}=3.85$ y ro $=20.26$ como se muestra en la Tabla 3 .

Tabla 3: Zonas bradfordianas de las revistas y artículos.

\begin{tabular}{lcccc} 
Zonas & Revistas & \% revistas & Artículos & \% artículos \\
\hline Zona 1 & 11 & 2.4 & 419 & 29.9 \\
Zona 2 & 65 & 14.1 & 427 & 30.5 \\
Zona 3 & 386 & 83.5 & 556 & 39.6 \\
\hline Total & $\mathbf{4 6 2}$ & $\mathbf{1 0 0 . 0}$ & $\mathbf{1 4 0 2}$ & $\mathbf{1 0 0 . 0}$ \\
\hline
\end{tabular}

Fuente: datos de la investigación. 
Aproximadamente un tercio de los artículos (30\%) fueron publicados prioritariamente en 10 revistas nacionales y una extranjera. Por medio de la revista Ciência da Informação se ha diseminado el mayor número de artículos (9\%) que aplican las técnicas bibliométricas. La revista Scientometrics, Encontros Bibli y Perspectivas em Ciência da Informação son las revistas con las siguientes preferencias de los autores (Tabla 4).

Tabla 4: Revistas del núcleo Bradfordiano.

\begin{tabular}{lc}
\hline Título de la Revista & No.de artículos \\
\hline Ciência da Informação & 123 \\
Scientometrics & 53 \\
Encontros Bibli & 48 \\
Perspectivas em Ciência da Informação & 44 \\
Transinformação & 33 \\
Em Questão & 24 \\
Revista de Administração Contemporânea & 23 \\
DataGramaZero & 20 \\
Informação \& Sociedade: Estudos & 18 \\
RAE-Revista de Administração de Empresas & 18 \\
Informação \& Informação & 15 \\
Revista Brasileira de Biblioteconomia e Documentação & 15 \\
PontodeAcesso & 15 \\
\hline
\end{tabular}

Fuente: datos de la investigación.

A estas onces revistas se le agregaron las dos últimas listadas en esta tabla porque presentan el mismo número de artículos que la incluida en el núcleo Bradfordiano. Estas trece revistas (Tabla 4) son las preferidas por los investigadores para divulgar los resultados de sus investigaciones. Diez de estas revistas están dedicadas al campo de la bibliotecología y ciencias de la información, una al campo de la cienciometría y dos al campo de administración.

$36.5 \%$ de los documentos fueron publicados como ponencias presentadas en congresos y eventos similares (mesas redondas, encuentros científicos, workshops, etc.). Para identificar los eventos preferidos para presentar los resultados de las investigaciones se usó nuevamente el método de división zonal de la ley de Bradford propuesto por Egghe (1990). Esta división se estimó con $\mathrm{A}=840 ; \mathrm{P}=3$ y $\mathrm{T}=183$ dando como resultado $\mathrm{y}_{\mathrm{o}}=280 ; \mathrm{k}=6.0 \mathrm{y} \mathrm{r}_{\mathrm{o}}=4.26$ (Tabla 5).

Tabla 5: División zonal Bradfordiano de congresos.

\begin{tabular}{lcccc}
\hline Zonas & Congresos & \% Congresos & Ponencias & \% Ponencias \\
\hline Zona 1 & 4 & 2.2 & 285 & 33.9 \\
Zona 2 & 26 & 14.2 & 331 & 39.4 \\
Zona 3 & 153 & 83.6 & 224 & 26.7 \\
\hline Total & $\mathbf{1 8 3}$ & $\mathbf{1 0 0 . 0}$ & $\mathbf{8 4 0}$ & $\mathbf{1 0 0 . 0}$ \\
\hline
\end{tabular}

Fuente: datos de la investigación.

$34 \%$ de los documentos fueron presentados en los 4 primeros congresos que se listan en la Tabla 6. Los 13 congresos listados fueron empleados más frecuentemente para intercambiar opiniones sobre las investigaciones en curso o concluidos tanto que acumulan juntas el $62 \%$ de los documentos presentados como ponencias en congresos. El 38\% de los documentos restantes fueron presentados en 178 diversos congresos nacionales e internacionales, pero solamente divulgaron entre 1 a 8 documentos. De estos 13 eventos, 4 son del campo de la bibliotecología y ciencia de la información, 4 de administración, 3 de gestión del conocimiento o ingeniería de la producción y 2 de contabilidad y costos. 
Tabla 6: Congresos más utilizados para divulgación de resultados.

\begin{tabular}{lc}
\hline Nombre del evento & No. de ponencias \\
\hline ENANCIB & 120 \\
Encontro Brasileiro de Bibliometria e Cientometria & 92 \\
EnANPAD: Encontro Nacional da ANPAD & 73 \\
SEMEAD: Seminários em Administração & 57 \\
Seminário Nacional de Bibliotecas Universitárias & 47 \\
Congresso Brasileiro de Custos & 33 \\
Congresso USP de Contabilidade e Controladoria & 20 \\
Encontro Nacional de Engenharia de Produção & 18 \\
Congresso Brasileiro de Biblioteconomia e Documentação & 13 \\
Encontro de Ensino e Pesquisa em Administração e Contabilidade & 13 \\
Simpósio de Excelência em Gestão e Tecnologia & 12 \\
Simpósio de Gestão de Inovação Tecnológica & 12 \\
Encontro da Divisão de Administração Pública e Governança & 10 \\
\hline
\end{tabular}

Fuente: datos de la investigación.

Para identificar la elite de los autores más productivos se usó la "ley de la raíz cuadrada" de Price (1963), quien afirma que esta raíz cuadrada de la población total de autores "inmediatamente produce un método objetivo para separar los mayores de los menores contribuyentes. Uno puede establecer un límite y decir que la mitad del trabajo es hecho por aquellos con más de 10 artículos, o que el número de los grandes productores parece ser de la misma orden de magnitud que la raíz cuadrada del número total de autores" (Price, 1963:46). Por este método se estimó un total de 57.6 autores. La cifra más cercana a esta cantidad son los 55 primeros autores listados en el Anexo D. Para cada uno de estos autores se estimó su respectivo "coeficiente de colaboración" (CC) con el método propuesto por Ajiferuke; Burell e Tague (1988) así como también su tasa de productividad.

Utilizando BibExcel se estableció también la red de colaboración de estos 55 autores de mayor productividad. El resultado puede observarse en la Figura 1. Se puede prestar atención a las figuras centrales en la red de colaboradores. Por ejemplo, M.C.P.I. Hagashi; L.I.L. de Faria y R.N.M. dos Santos. Otro grupo liderado por E.F.T. de Oliveira. También otro liderado por I. R.C. Stumpf y S. E. Caregnato. Un grupo casi aislado liderado por L. Rossoni y otro por S. R. Ensslin. El resto de autores son colaboradores ocasionales. 


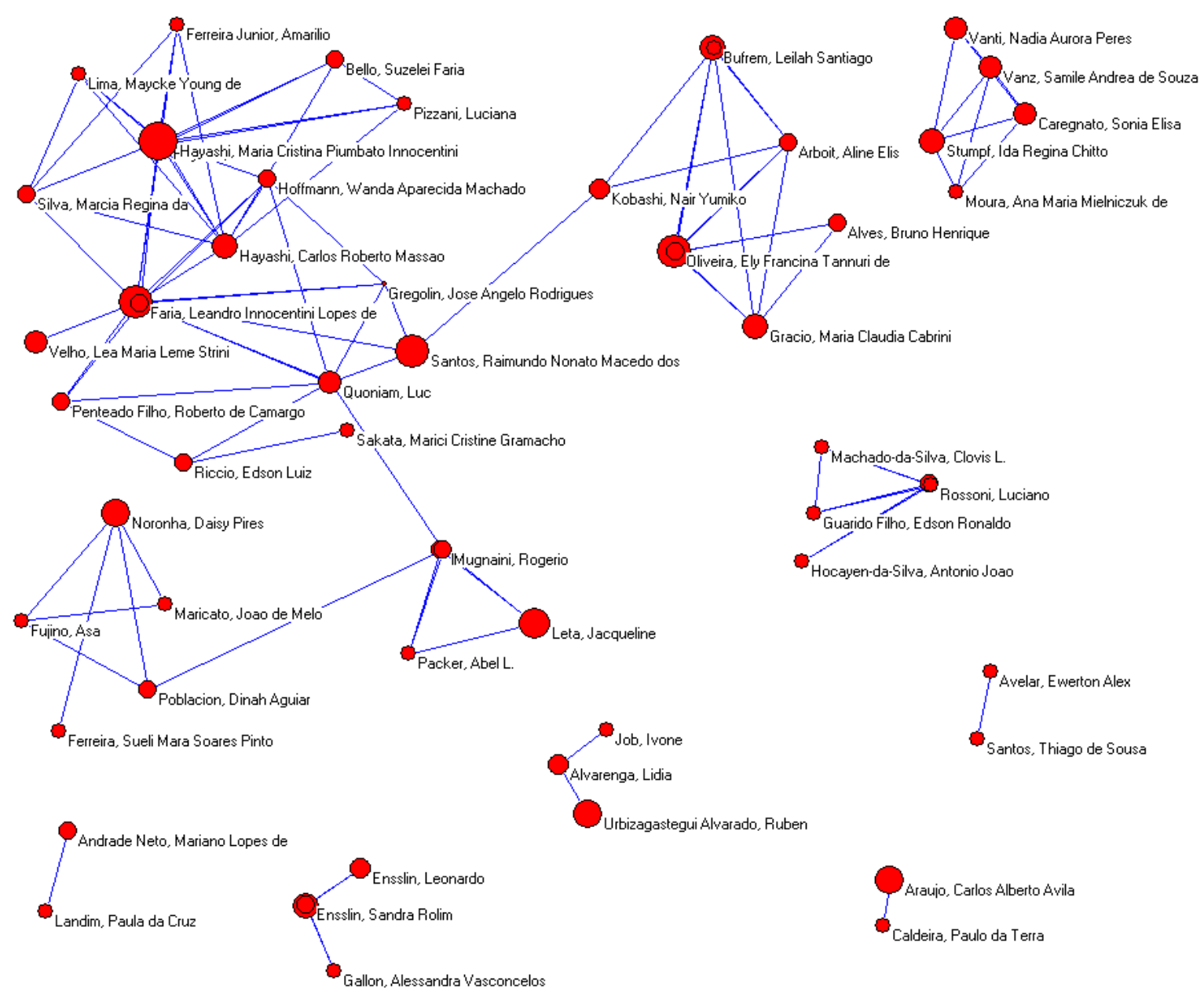

Figura 1: Red de colaboración de la elite de productores en metrías en el Brasil.

Fuente: datos de la investigación.

Como era de esperarse, estos grandes productores tienen doctorado, son profesores en universidades, muchos son miembros del consejo editorial de las revistas en las que publican, y algunas tienen cargo de dirección en bibliotecas especializadas o universitarias, orientan alumnos en la elaboración de tesis y disertaciones, tienen alumnos que actúan como ayudantes en la recopilación de información para investigación y en muchos casos las publicaciones son avances o resultados de las tesis y disertaciones elaboradas por los alumnos dirigidos pero publicados en colaboración (coactada) con los profesores orientadores de las mismas. Pero esto no es novedad, ya había sido estudiado y predicho por Urbizagástegui (2008). La figura 2 muestra la red de colaboración de la investigadora más productiva en los estudios métricos en el Brasil. Para la elaboración del gráfico se consideraron solamente las colaboraciones de los autores en 3 y más documentos. 


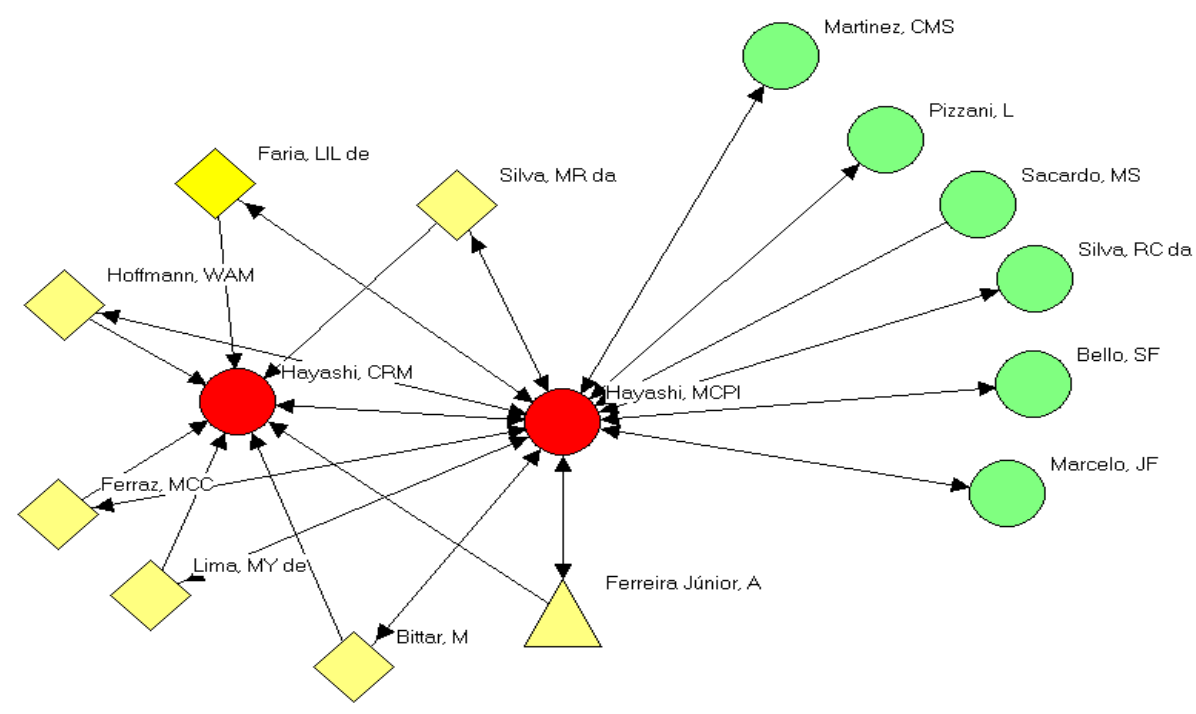

Figura 2: Grupo de colaboración liderado por M.C.P. I. Hayashi. Fuente: datos de la investigación.

\section{DISCUSIÓN Y CONCLUSIÓN}

Las diferencias de resultados con las investigaciones anteriores (VANZ, 2003; LIMA et al., 2011; GRÁCIO, OLIVEIRA, 2012) se deben principalmente a la amplitud temporal de las investigaciones. Mientras que esas investigadoras centraron su atención en periodos cortos, el presente estudio abarca un periodo de 40 años (19732012). Algunas de esas estudiosas también redujeron su investigación a las publicaciones de una única revista, o entre cinco y diez revistas del campo de la bibliotecología y ciencias de la información, otras solamente a tesis y disertaciones; mientras que esta investigación recopiló todas las publicaciones realizadas en el país y las realizadas por brasileños en el exterior. Es decir, la cobertura es mucho más amplia y representativa de la práctica científica brasileña.

Otra de las diferencias se relaciona a la forma de búsqueda de las publicaciones: algunas autoras no indican las palabras claves usadas para la recuperación (VANZ, 2003) de los documentos. Otras redujeron sus términos de búsqueda a cinco palabras claves (LIMA et al., 2011), todavía otras a una combinación de 17 palabras claves (GRÁCIO, OLIVEIRA, 2012). Lógicamente, eso también produjo diferencias tanto en la cantidad de documentos recuperados como en el número de autores productores de esos documentos identificados. Por ejemplo, Vanz (2003) encontró apenas 40 autores, cuatro de ellos publicando antes de la década de los $90 \mathrm{~s}$. Este pequeño número de autores la indujo a afirmar "la inexistencia de investigaciones sobre la temática, haciendo que los autores publiquen ocasionalmente sobre este asunto" (VANZ, 2003, p. 13). Otro dato que comprobaría la inexistencia de investigación formal sobre bibliometría en el Brasil sería el hecho de que dos de los cuatro autores más productivos eran extranjeros (VANZ, 2003, p. 14). Sin embargo, hay que tener presente que la autora se está refiriendo a datos recogidos de una única revista y a la década de los 90s. Igualmente, Lima et al. (2011) identificaron 151 artículos producidos por 203 investigadores, con 20 de ellos con por lo menos tres publicaciones, pero cinco de los autores considerados como grandes productores no hacen parte de ese grupo de "grandes productores" en esta investigación. También Grácio e Oliveira (2012) identificaron 31 investigadores con por lo menos 3 artículos publicados, es decir, grandes productores. En esta investigación se identificaron 55 investigadores como parte de la elite de grandes productores sobre este tópico en el Brasil (Anexo D) con diez y más documentos publicados por cada uno de ellos/ellas. Nuevamente, los resultados de esta 
investigación contradicen esas afirmaciones pues los 2,300 documentos encontrados fueron producidos por 3,320 investigadores y los autores extranjeros que publicaron en el Brasil no son ni los más productivos ni están incluidos en el frente de investigación (excepto uno que declara al Brasil como su patria adoptiva). Igualmente, Vanz (2003) afirma la inexistencia de grupos de investigación sobre este asunto pero nuevamente los resultados de esta investigación contradicen esas afirmaciones pues existen grupos de investigación consolidados sobre bibliometría en el Brasil (Ver Figuras 1 y 2).

Resumiendo, los medios de comunicación más usados para comunicar los resultados de las investigaciones en la bibliometría brasileña son los artículos publicados en revistas académicas. Los investigadores se sirven básicamente de la lengua portuguesa que es el idioma nativo del país. De un total de 462 revistas utilizadas para diseminar los resultados de las investigaciones en bibliometría, se identificó un núcleo de 13 revistas como las preferidas por los investigadores brasileños. Estas revistas no son especializadas en bibliometría, pero el volumen de producción es tan elevado que ya es posible predecir que pronto surgirá una revista especializada en esta área en el Brasil. Por ahora ya existe un evento especializado en bibliometría, informetría, y cienciometría brasileña que se realiza cada dos años.

Se identificaron también 183 eventos diferentes en los que fueron presentadas 840 ponencias. Trece de esos eventos son centrales para la diseminación de los resultados de las investigaciones. Muchos de estos eventos son de diferentes campos del conocimiento con predominancia de la bibliotecología y ciencia de la información, administración, contabilidad, costos, gestión y organización del conocimiento. Se identificó una élite de 55 productores pero estos no son responsables de la mitad de las contribuciones. Estos autores colaboran entre si y tienen una alta tasa de colaboración y productividad.

\section{BIBLIOGRAFÍA}

AJIFERUKE, Isola; BURREL, Q.; TAGUE, J. Collaborative coefficient: a single measure of the degree of collaboration in research. Scientometrics, v. 14, n. 5/6, p. 421-433, 1988.

ARAÚJO, Ronaldo Ferreira de; ALVARENGA, Lídia. A bibliometria na pesquisa científica da pós-graduação Brasileira de 1987 a 2007. Encontros Bibli: Revista Eletrônica de Biblioteconomia e Ciência da Informação, v. 16, n. 31, p. 51-70, 2011.

AZAMBUJA, Ana Paula de Almeida. A bibliometria nos periódicos de ciência da informação no Brasil. Rio Grande, Universidade Federal do Rio Grande, Instituto de Ciências Humanas e da Informação, Curso de Biblioteconomia, 2011. Trabalho de conclusão de curso de biblioteconomia.

EGGHE, LEO. Applications of the theory of Bradford's law to the calculation of Leimkuhler's Law to the completion of bibliographies. Journal of the American Society for Information Science, v. 41, n. 7, p. 469-492, 1990.

FONTES, Augusto César Oliveira. Os estudos métricos no Brasil: uma análise a partir das revistas eletrônicas de ciência da informação. Trabalho de Conclusão de Curso apresentado ao Departamento de Biblioteconomia e Documentação da ECA/USP como cumprimento para obtenção do título de bacharel em Biblioteconomia. São Paulo, Brasil, 2008.

GRÁCIO, Maria Claudia Cabrini; OLIVEIRA, Ely Francina Tannuri de. A inserção e o impacto internacional da pesquisa brasileira em "estudos métricos": uma análise na base Scopus. Tendências da Pesquisa Brasileira em Ciência da Informação, v. 5, n. 1, p. 71-113, 2012. 
LETA, Jacqueline. Brazilian scientometrics: from little to big?. ISSI Newsletter, v 8, n. 3, p. 34$37,2012$.

LIMA, Lidyane Silva; SOARES, Carolina Ferreira; OLIVEIRA, Ely Francina Tannuri de. Investigação da produção científica no tema "estudos métricos" na Base de Dados Brapci: uma análise bibliométrica". Revista EDICIC, v. 1, n. 4, p. 299-310, 2011.

MACHADO, Raymundo das Neves; PINTO, Emaunel Vieira. Mapeamento da produção científica em bibliometria (1990-2004). In ENANCIB: Encontro Nacional de Pesquisa em Ciência da Informação, 6., 2005, Florianópolis, SC. Anais do VI Enancib. Florianópolis: Universidade Federal de Santa Catarina, 2005.

MACHADO, Raymundo das Neves. Análise cientométrica dos estudos bibliométricos publicados em periódicos da área de biblioteconomia e ciência da informação (1990-2005). Perspectivas em Ciência da Informação, v. 12, n. 3, p.2-20, 2007.

MATTOS, Ana Maria; JOB, Ivone. A produção científica brasileira no periódico Scientometrics de 1978 até 2006. Encontros Bibli: Revista Eletrônica de Biblioteconomia e Ciência da Informação, v. 13, n. 26, pp. 47-61, 2008.

MENEGHINI, Rogério; PACKER, Abel L. The extent of multidisciplinary authorship of articles of scientometrics and bibliometrics in Brazil. Interciencia, v. 35, n. 7, p. 510-514, 2010.

MUGNAINI, Rogério; JANNUZZI, Paulo de Martino; QUONIAM, Luc. Indicadores bibliométricos da produção científica brasileira: uma análise a partir da base Pascal. Ciência da Informação, v. 33, n. 2, p. 123-131, 2004.

PINHEIRO, Lena Vania Ribeiro; SILVA, Giselle Santos. Cartografia histórica e conceitual da bibliometria / informetria no Brasil. In Conferencia Ibero-Americana de Publicacoes Eletronicas no Contexto da Comunicacao Cientifica, II CIPECC., 2008, Rio de Janeiro, RJ. Rio de Janeiro, RJ, Brasil, 17 a 21 de novembro de 2008.

PRICE, John Derek de Solla. Little science, big science. New York: Columbia University Press, 1963.

PRICE, John Derek de Solla. Networks of scientific papers. Science, v. 149, n. 3683, p. 510-515, 1965.

PRICE, John Derek de Solla. The structure of publication in science and technology. In: GRUBER, William H.; MARQUIS, Donald G. (Ed.). MIT Conference on the Human Factor in the Transfer of Technology, 1966, Endicott House. Factors in the transfer of technology. Cambridge, MIT Press, [1969]. p. 91-104.

PRICE, John Derek de Solla. Some remarks on elitism in information and the invisible college phenomenon in science. Journal of the American Society for Information Science, v. 22, n. 2, p. 74-75, 1971.

PRICE, John Derek de Solla. The productivity of research cientists. In: Encyclopaedia Britannica: yearbook of science and the future. Chicago: Encyclopaedia Britannica, Inc., 1975. p. 409-421.

SANTOS, Raimundo Nonato Macedo dos. Indicadores estratégicos em ciência e tecnologia: refletindo a sua prática como dispositivo de inclusão/exclusão. Transinformação, v. 15, n. 3, p. 129-140, 2003.

URBIZAGÁSTEGUI ALVARADO, Rubén. A bibliometria no Brasil. Ciência da Informação, v. 13, n. 2, p. 91-105, 1984. 
URBIZAGÁSTEGUI ALVARADO, Rubén. A cienciometria como um campo cientifico. Informação \& Sociedade: Estudos, v. 20, n. 3, p. 41-62, 2010.

VANZ, Samile Andréa de Souza. A Bibliometria No Brasil: Analise Tematica Das Publicacoes Do Periodico Ciencia da Informação (1972-2002). In ENANCIB: Encontro Nacional de Pesquisa em Ciência da Informação (5to. : Belo Horizonte : 2003). Anais do ENANCIB. Belo Horizonte: UFMG, 2003 Belo Horizonte, Minas Gerais, de 10 a 14 de novembro de 2003: ANCIB, 2003.

Editores do artigo: Adilson Luiz Pinto, Rafaela Paula Schmitz e Enrique Muriel-Torrado

\section{ANEXO A}

Autores que han estudiado la bibliometría en el Brasil

\begin{tabular}{|c|c|c|c|c|}
\hline Años & AUTORES & FUENTES USADAS & PERIODO & RESULTADOS \\
\hline 1984 & Urbizagástegui & Documentos en general & $1972-1983$ & 78 documentos \\
\hline 2003 & Vanz & Ciencia da Informação & $1972-2002$ & Pocas Publicaciones \\
\hline 2005 & Machado; Pinto & 5 revistas de BCI & 1990-2004 & 27 artículos \\
\hline 2007 & Machado & 5 revistas de $\mathrm{BCI}$ & $1990-2005$ & 31 artículos; 19 autores \\
\hline 2008 & Fontes & $\begin{array}{l}10 \text { revistas de BCI; } \\
\text { Dedalus BD da USP; } \\
\text { Scielo CAPES }\end{array}$ & Hasta 2007 & 84 Artículos \\
\hline 2008 & Pinheiro; Costa & Tesis IBICT & $1972-2008$ & 57 tesis y disertaciones \\
\hline 2008 & Matos; Job & Revista Scientometrics & $1978-2006$ & 34 artículos \\
\hline 2010 & Menegenni; Parker & $\begin{array}{l}\text { Web of Science; Google } \\
\text { Scholar; SciELO Brasil; } \\
\text { Plataforma Lattes }\end{array}$ & $1990-2006$ & 197 documentos \\
\hline 2011 & Araujo; Alvarenga & $\begin{array}{c}\text { Banco de Tesis da } \\
\text { CAPES }\end{array}$ & $1987-2007$ & $\begin{array}{l}82 \text { tesis y disertaciones; } 23 \\
\text { instituciones de enseñanza }\end{array}$ \\
\hline 2011 & Azambuja & 8 Revistas de BCI & $2006-2011$ & 33 artículos \\
\hline 2011 & Lima, et al. & BRAPCI & $1991-2011$ & 151 artículos; 203 investigadores \\
\hline 2012 & Gracio; Oliveira & Scopus & $1984-2012$ & 263 artículos; 649 investigadores \\
\hline 2012 & Leta & Scientometrics & ------.-- & Recuento teórico \\
\hline
\end{tabular}

\section{ANEXO B}

Términos de búsqueda

\begin{tabular}{l}
\hline Brasil \\
Índice h \\
Elitismo \\
Frente de investigación \\
Regla $80 / 20$ \\
Obsolescencia de la literatura \\
Crecimiento de la literatura \\
Vida media \\
Teoría epidémica \\
Visibilidad \\
Índice de Pratt \\
Índice de Price \\
Índice de inmediatismo \\
Ley de Price \\
Indicadores bibliométricos \\
Indicadores cienciométricos \\
Ley de Goffman \\
Ley de Bradford \\
Ley de Lotka \\
Ley de Zipf \\
Punto de transición
\end{tabular}


Colegios invisibles

Factor de impacto

Factor de inmediatismo

Análisis de citas

Acoplamiento bibliográfico

Co-citación

Redes sociales

Co-autoría

Colaboración científica

Índice de colaboración

Circulación de la colección

Núcleo básico de periódicos

Indicadores en ciencia y tecnología

Bibliometría

Cienciometría

Informetría

Patentometría

Arquivometría

Bio-bibliometría

Webometría

Sitometría

Netometría

\section{ANEXO C}

\section{Bases de Datos Bibliográficos y Portales consultadas}

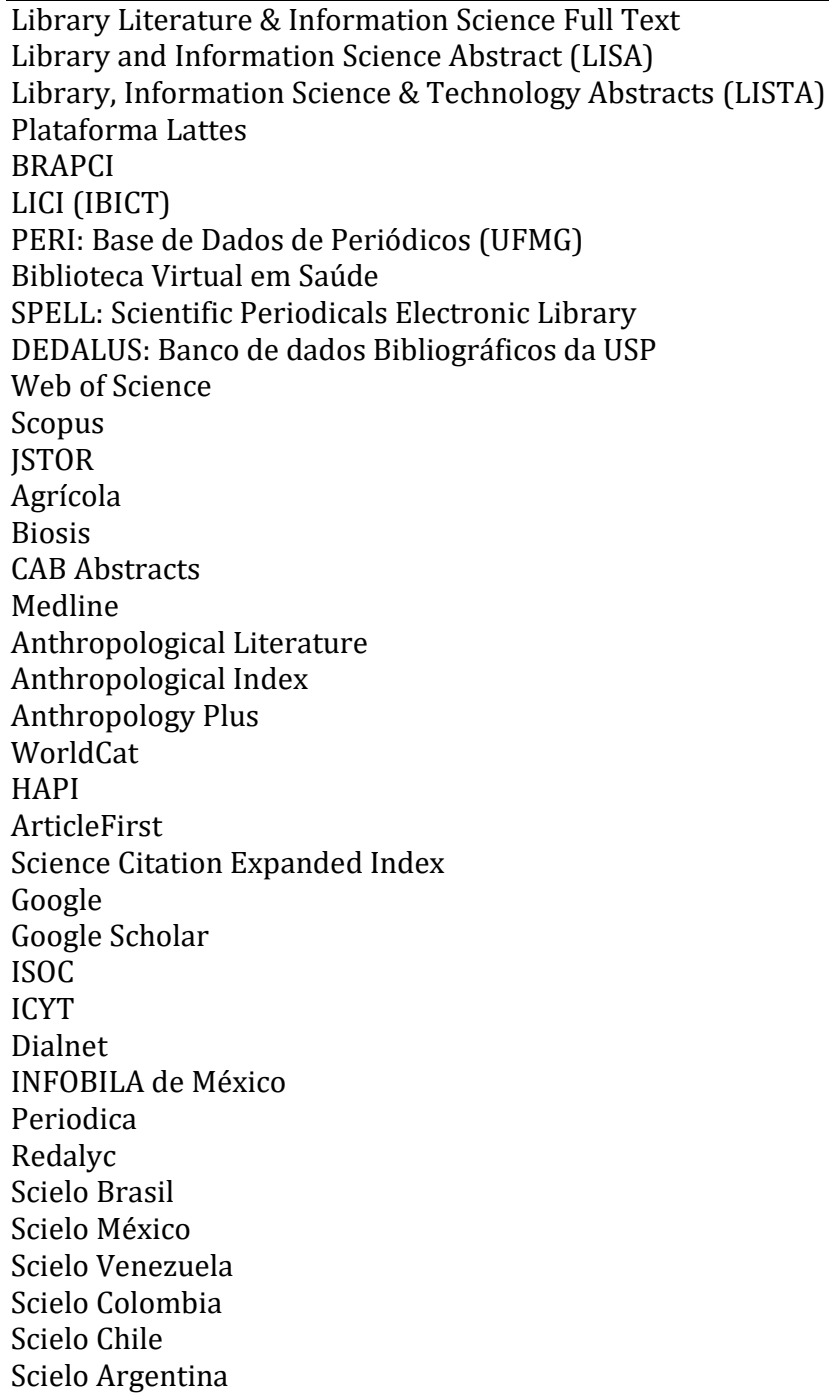


Scielo Bolivia

Portal del Ricyt

Biblioteca Virtual en Salud del Brasil

Biblioteca Virtual de la Universidad de São Paulo

y otras 120 bases de datos bibliográficas existentes en la biblioteca de ciencias de la Universidad de California en Riverside.

\section{ANEXO D}

Autores más productivos, coeficiente de colaboración (CC) y tasa de producción (TP)

\begin{tabular}{|c|c|c|c|}
\hline Autores & Número de docs producidos & $\mathbf{C C}$ & TP \\
\hline Hayashi, Maria Cristina Piumbato Innocentini & 65 & 0.64 & 7.2 \\
\hline Faria, Leandro Innocentini Lopes de & 56 & 0.67 & 3.7 \\
\hline Oliveira, Ely Francina Tannuri de & 48 & 0.58 & 6.9 \\
\hline Santos, Raimundo Nonato Macedo dos & 46 & 0.59 & 2.5 \\
\hline Ensslin, Sandra Rolim & 39 & 0.69 & 6.5 \\
\hline Leta, Jacqueline & 39 & 0.54 & 2.3 \\
\hline Noronha, Daisy Pires & 34 & 0.57 & 1.0 \\
\hline Hayashi, Carlos Roberto Massao & 32 & 0.72 & 3.5 \\
\hline Urbizagástegui Alvarado, Rubén & 30 & 0.07 & 1.0 \\
\hline Rossoni, Luciano & 29 & 0.64 & 0.6 \\
\hline Bufrem, Leilah Santiago & 28 & 0.56 & 2.4 \\
\hline Grácio, Maria Cláudia Cabrini & 27 & 0.57 & 5.4 \\
\hline Vanz, Samile Andréa de Souza & 27 & 0.55 & 2.5 \\
\hline Stumpf, Ida Regina Chittó & 25 & 0.61 & 1.9 \\
\hline Vanti, Nadia Aurora Peres & 23 & 0.30 & 1.8 \\
\hline Gregolin, José Angelo Rodrigues & 22 & 0.75 & 1.5 \\
\hline Caregnato, Sônia Elisa & 22 & 0.52 & 2.2 \\
\hline Velho, Lea Maria Leme Strini & 21 & 0.74 & 1.1 \\
\hline Ensslin, Leonardo & 20 & 0.72 & 3.3 \\
\hline Quoniam, Luc & 20 & 0.73 & 1.1 \\
\hline Mugnaini, Rogério & 18 & 0.50 & 1.6 \\
\hline Araújo, Carlos Alberto Ávila & 18 & 0.44 & 1.8 \\
\hline Beuren, Ilse Maria & 17 & 0.60 & 2.2 \\
\hline Kobashi, Nair Yumiko & 17 & 0.60 & 2.1 \\
\hline Ribeiro, Henrique César Melo & 17 & 0.57 & 5.6 \\
\hline Bello, Suzelei Faria & 17 & 0.64 & 3.4 \\
\hline Hoffmann, Wanda Aparecida Machado & 16 & 0.77 & 1.1 \\
\hline Walter, Silvana Anita & 16 & 0.71 & 3.2 \\
\hline Población, Dinah Aguiar & 16 & 0.52 & 1.1 \\
\hline Pinto, Adilson Luiz & 15 & 0.43 & 2.5 \\
\hline Silva, Márcia Regina da & 14 & 0.62 & 1.9 \\
\hline Witter, Geraldina Porto & 14 & 0.30 & 0.6 \\
\hline Mattos, Ana Maria & 14 & 0.49 & 1.8 \\
\hline Meneghini, Rogério & 14 & 0.34 & 0.6 \\
\hline Mueller, Suzana Pinheiro Machado & 14 & 0.32 & 0.6 \\
\hline Espejo, Márcia Maria dos Santos Bortolocci & 14 & 0.74 & 2.8 \\
\hline Guarido Filho, Edson Ronaldo & 14 & 0.65 & 2.3 \\
\hline Cruz, Ana Paula Capuano da & 12 & 0.75 & 1.7 \\
\hline Hocayen-da-Silva, Antônio João & 12 & 0.61 & 1.7 \\
\hline Penteado Filho, Roberto de Camargo & 12 & 0.61 & 1.7 \\
\hline Pizzani, Luciana & 12 & 0.69 & 2.0 \\
\hline Oliveira, Robson Ramos & 12 & 0.67 & 2.4 \\
\hline Silva, Fábio Mascarenhas e & 12 & 0.66 & 1.1 \\
\hline Riccio, Edson Luiz & 12 & 0.73 & 0.9 \\
\hline Alvarenga, Lídia & 11 & 0.35 & 0.4 \\
\hline Rummler, Guido & 11 & 0.18 & 1.2 \\
\hline Machado-da-Silva, Clóvis L. & 11 & 0.63 & 0.5 \\
\hline Ferreira Júnior, Amarílio & 11 & 0.70 & 1.4 \\
\hline Caldeira, Paulo da Terra & 10 & 0.38 & 0.3 \\
\hline Silveira, Murilo Artur Araújo da & 10 & 0.61 & 1.4 \\
\hline Brambilla, Sônia Domingues Santos & 10 & 0.57 & 1.2 \\
\hline Lima, Maycke Young de & 10 & 0.62 & 1.4 \\
\hline Santos, Jane Lucia Silva & 10 & 0.73 & 1.7 \\
\hline Martins, Gilberto de Andrade & 10 & 0.55 & 1.1 \\
\hline
\end{tabular}


